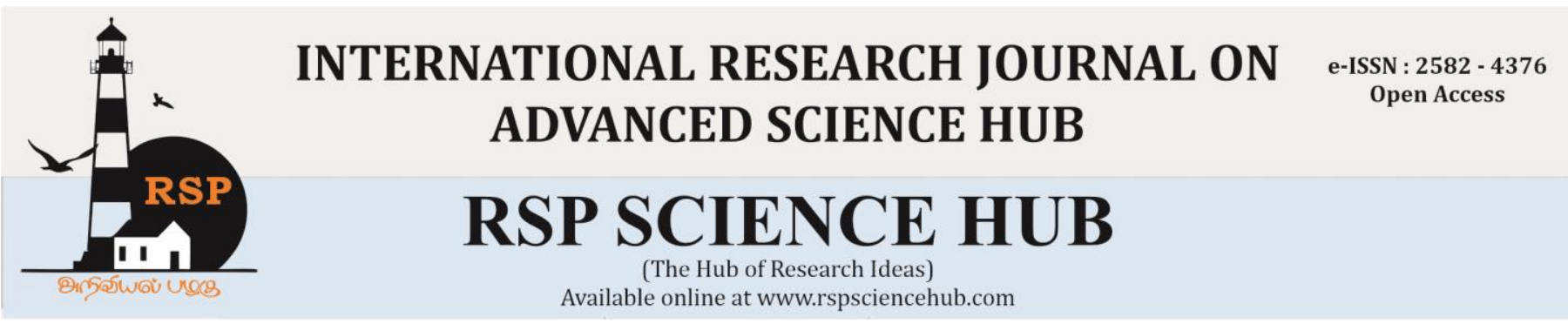

\title{
Development of Voice Operated Exoskeleton Arm Using Solar Power
}

\author{
Chalana. $V^{1}$, Christy Rockey ${ }^{2}$, Shifa Fathima. $N^{3}$, AlMubashir.P. $M^{4}$ \\ 1,2,3,4UG Scholar, Department of Biomedical Engineering, Karpagam Academy of Higher Education,
}

Tamilnadu, India.

\begin{abstract}
Exoskeleton are as of now being investigated for help of those with weak limbs. A great deal of paralyzed individuals with motor disabilities are confronting challenges. This paper is to develop a model undertaking of voice worked exoskeleton arm utilizing solar power going about as a help gadget for paralyzed individuals. It audits the upper exoskeleton arm with expanded degrees of freedom (DOF), voice commands and solar power. The exoskeleton arm makes no falsification of supplanting the lost arm, however attempts to supplant a few capacities that were lost. The gadget can be worn according to will and can be evacuated when not needed. A nonattractive material is utilized for the manufacture and will attempt to overcome the confinements in decreased expense and weight.
\end{abstract}

Keywords: exoskeleton arm, solar power, degrees of freedom, voice operated

\section{Introduction}

The researches in the field of Rehabilitation is continuing from decades and is still going on .The recent examinations by the World Health Organisation (WHO) says that about $15 \%$ of the total population experiences a type of inability, in that more than half cannot bear the cost of health care systems[2-6]. Due to different political reasons, the general and appendage brokenness patients are expanding. There are more than 10 million disabled all over, out of which $30 \%$ are arm disabled persons. Many of the people as been diagnosed with different types of injuries and new cases have been diagnosing still. Almost $50 \%$ of these cases bring about some loss of sensation or movement to the arms and hands[1]. The growth and use of exoskeleton arm is considered as the major remedy for this situation where the muscles does not receive any signals from the central nervous system.An exoskeleton consists of structure and joints that are very much similar to the human anatomy. It will adjust with an individual and such that the physical contact between the administrator and the exoskeleton empowers an immediate exchange of mechanical force and data signals[15-19]. Similarly it gives a compelling interface between the mechanical structure and the upper limb of a person. The ability to move is an important and the basic need in the daily life of every person .Therefore a motor disability can diminishes altogether the personal satisfaction of the individuals[22-25] .The disabled person will lose his independence also .He or she need to depend on others to get their needs done The advances in the technology has been concentrating on the recovery of the movement of upper limb. The patients requiring for physical rehabilitation of upper limb has been increasing considerably[7-11] .This situation give rise to the increasing number of specialists and caretakers for helping the physically debilitated people at their home, which will become a issue in the future. The major objective is to create an exoskeleton that enables to the movement of the upper limb for both the motions of the elbow 
,forearm and shoulder .But these devices may or may not be reasonably affordable and household use[8-10].A few ways to deal with re-establish the usefulness of the furthest point .Many works has to be done to focus on the upper limb rehabilitation .More 250,000 people have been diagnosed with injuries in spinal cord and new cases has been diagnosed every year[21] .To find a solution an exoskeleton arm has to be introduced .Exoskeleton robots are generally investigated in the regions identified with recovery .Assistive robotics and automated recovery are getting progressively imperative to physically feeble and old population .Thus the exoskeleton will improve the quality of the life of the people who depends on others for their daily life activities [12]. The concept of exoskeleton has been proposed in 1883 ,the first exoskeleton was designed in 1936 ."Hardiman" in 1961 developed a whole body exoskeleton robot using several hydraulic actuators[11-14] .The exoskeleton can be very helpful for the paralysed people and the old age people. Typically treatment for these conditions depends somewhat on manipulative physiotherapy methodology which by their very nature are incredibly labour intensive requiring elevated levels of balanced consideration from skilled medical. staff. This made us a thought to develop a voice operated exoskeleton arm using solar power as a supporting aid to the paralyzed people[25].Even though there many attempts been taken so far, still researches are going on in improving the life of the people and making it so easy. In this paper we are trying to develop a prototype model of exoskeleton by overcoming the existing limitations and the use of solar power in addition will make it more adaptable. The fabrication is done by incorporating a microcontroller along with the speech module[1620]. The received voice is identified and the voice signals are quantified and sampled.

\section{Goals And Requirements}

We set our goals and requirements for designing the exoskeleton arm is as follows:

- To develop a prototype project of voice operated exoskeleton arm by using solar power.

- To develop an interface between solar and exoskeleton arm.

- To increase the degrees of freedom of the existing device.
- To reduce the size and weight of the device to prevent the fatigue of the user.

- To preserve the comfort of the user and to prevent any injuries or discomfort.

- To eliminate the external voice interfaces.

- To add solar power as the additional power source.

\section{Mechanical Design}

The mechanical design of the proposed methodology consists of Aluminium framework Mechanical design has been designed with the criteria such as with proper weight ,easy replacement of parts and maintenance and easy assembly .The mechanical system is able to adjust its size and is portable .It is designed with a three sections including shoulder ,elbow and wrist[13]. Exoskeleton transmits torques to the joints by means of actuators allocated in its mechanical structure. The light weight model provides low mass and inertia to the system. The actual human arm comprises of 3 dof in the shoulder, $2 \mathrm{D}$ in the elbow .Appropriate antagonistic torque has been produced by providing joint motion on the exoskeleton .Various hardware tools are embedded with mechanical model. It includes Arduino microcontroller ,relay ,fasteners, lead acid battery ,DC geared motor along with the blue tooth module.

\subsection{Arduino Microcontroller(AT mega 328p )}

AT mega328p is commonly used in autonomous system where simple, low cost, low powered microcontroller is required .It has 23 general purpose i/o lines and 32 general purpose working registers .Three flexible counters are provided with comparable modes. This operates between 1.8 to 5.5 volts .Maximum operating frequency is 20 Mhz.32 kb flash memory, $2 \mathrm{~kb}$ of SRAM, $1 \mathrm{~kb}$ of EEPROM is the memory capacity of the device. 2 external interrupts and 16 number of touch channels are the specific key parameters of the system[23] .A high relevant speed of 20 MIPS at $20 \mathrm{MHZ}$ is the operating performance of the micro controller .The device achieves 1 MIPS per MHZ by executing powerful instructions in a single clock cycle. The ATmega328p can be commonly replaced by the "pico Power". The single chip microcontroller ATmega328p is developed by Atmel in the mega AVR family[24]. The ATmega328p consist of a modified Harvard architecture 8 bit RISC processor core. 


\subsection{Aluminium Flats}

Phenomenon of passivation allows the aluminium to resist corrossion and its key feature low density .Oxides and sulphates are the compounds of aluminium .Aluminium is one of the most cheapest metal that is flexible for the fabrication purposes. The density of Aluminium is $2.70 \mathrm{~g} / \mathrm{cm}^{\wedge} 3$ which is $1 / 3$ that of steel. Its ductile and malleable property allows it to be easily drawn and extruded. Machining and casting can be easily done as it has low melting temperature of 660 degree celsius .Its light weight and paramagnetic nature is more convenient for the paralyzed people .It can be flexibly altered accordingly as per the need .It can be easily carried from one place to another place without any further requirements.

\subsection{Geared Motor}

Dc gear motor plays an important role in the main operation of the proposed exoskeleton arm.DC gear motor is the combination of gear box and motor[14] A gear head is added to a motor that increases the torque and reduces speed.The three parameters which defines the operating performance of the motor are speed, efficiency and torque .The main characteristic of DC motor is that when the applied voltage changes, load speed and starting torque does not change in proportion to the voltage.

\subsection{Relay}

Relay is used to control the speed of the exoskeleton arm to the specified direction as per requirement of the user. Relays acts as switch which works both electrically and electromechanically. The relay works on the principle of electromagnetic attraction. In the situation, when the relay circuit identifies a fault current, it will stimulate the electromagnetic field which produces temporary magnetic field[14]. The relay closes or opens the contacts to facilitate the working of the other electric control. The relay works on both electrically and mechanically. It includes a set of contacts and electromagnetic, performs the working of switching. It is mainly used in the circuits where independent low power signal is used to control the system .A driver current of 15 to $20 \mathrm{ma}$ is required for its operation.

\subsection{Lead Acid Battery(12 volt,1.3 ah)}

A 12 volt battery with the capacity of 1.3 ah is used in the proposed voice operated exoskeleton arm. These sealed maintenance free battery is suitable for various appliances. The self discharge rate of this is 3 to 20 percentage per month. Chargeable and dischargeable efficiency is 50-90 percentage .Energy density is $80-90 \mathrm{Wh} / \mathrm{L}$.Specify power is $180 \mathrm{~W} / \mathrm{kg}$.A low energy to weight ratio and low energy to volume ratio makes it to apply surge currents, which indicates that it has high power to weight ratio. As they are inexpensive, it has a wide variety of applications in the electronic and electromechanical systems. The nominal voltage of the lead acid battery is $2 \mathrm{~V}$ for each cell .It is found that the voltage varies from $1.8 \mathrm{v}$ loaded at full discharge to 2.10 volt in an open circuit at a full charge. Accumulation of excessive hydrogen and oxygen causes ignition in the internal environment.

3.6 HC 05 TTL Module

This module is used to communicate between the Arduino microcontrollers by using blue tooth with the functionality like phone and laptop .It has 2 operating modes :Data mode and command mode .Data mode is used to send and receive data from another blue tooth devices whereas command mode is used to change the default device settings .As it operates using serial port protocol, it makes easy for the blue tooth module to be connected with the microcontrollers .The operating current is $30 \mathrm{ma}$ with the operating voltage as $4 \mathrm{v}$ to $6 \mathrm{v}$.It can operate in Master ,Slave or Master/slave mode .It has the capability to work with serial communication and TTL compatible.

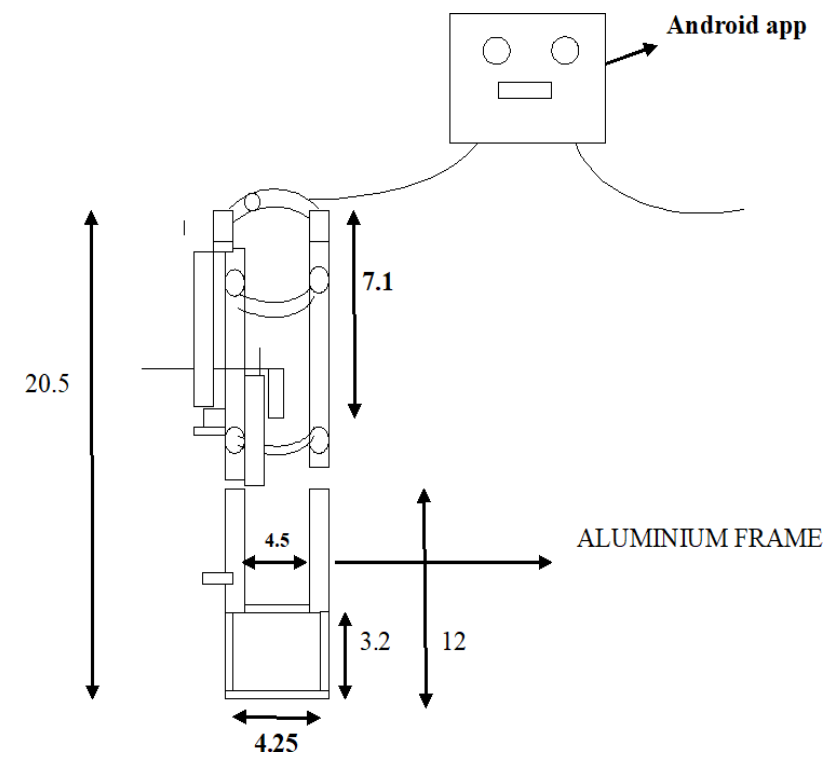

Fig.1. The image represents the 2D diagram of our exoskeleton arm. The diagram consists of measurements and design of the fabrication done using aluminium flats 


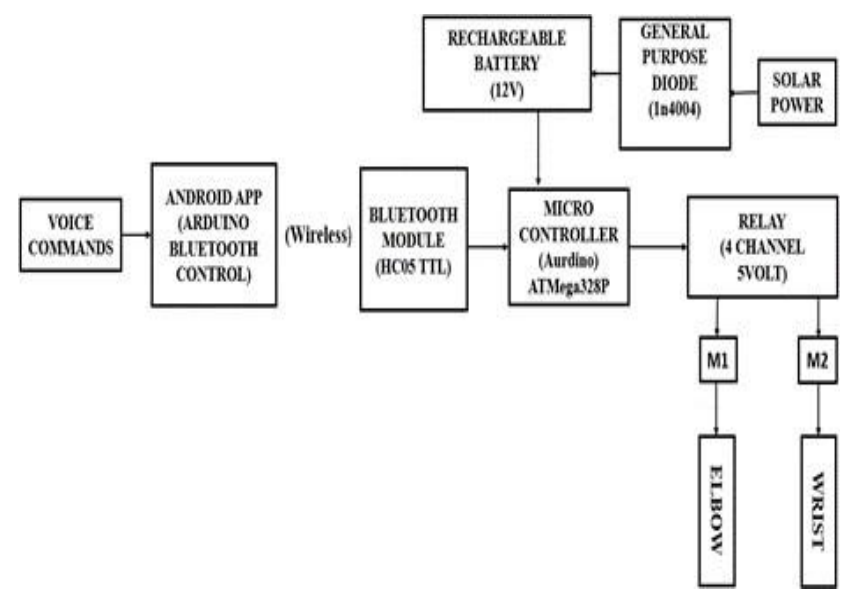

Fig.2. The block diagram representation includes the components and connections of our android based voice operated exoskeleton arm using solar power.

\section{Technical Specifications}

The aluminium flats used here is of $310 \mathrm{MPa}$ Ultimate Tensile Strength , $276 \mathrm{MPa}$ Tensile Yield Strength and of $69 \mathrm{GPa}$ Young's modulus of elasticity .Length of fore arm is $260 \mathrm{~mm}$ and bicep's length is $250 \mathrm{~mm}$. The fabrication is done by arc welding .The Arduino ATMega328P microcontroller has the operating voltage of $5 \mathrm{~V}$ and input voltage of 7-20V.It has 14 digital I/O pins and 6 analogue input pins. It is of low cost ,low power consumption and easy to program .It has a weight of $25 \mathrm{~g}$.The Jhonson DC geared motor of 12 Volts and $10 \mathrm{rpm}$ has the output torque range $5 \mathrm{~kg}-\mathrm{cm}$ to $7 \mathrm{~kg}-\mathrm{cm}$. It has a circular Dia with internal hole for coupling, 23mm shaft length .Load current is upto $9.5 \mathrm{a}(\max )$ and no-load current is $800 \mathrm{ma}(\max )$. The HC-05 Bluetooth module .It has 3.3 to 5 input output voltage .It has bluetooth stack inbuilt in it, can be directly connected to Android .The relay used here is 4 channel, 5 volt .It is TTL logic compatible .It is equipped with $3.1 \mathrm{~mm}$ screw holes for easy installation .The solar panel is of 17.1 Volts and 5Watts. The higher the voltage, the higher powerful is the panel. The battery used is a rechargeable battery of 12 volt , 10 ah .The battery can be used separately and also it can be used along with the solar panel. The voltage from the solar panel will be stored in the battery and can be used for the requirements.
Table.1. The table represents the each components included with their specifications.

\begin{tabular}{|c|c|c|}
\hline Sl.No & Components & Specifications \\
\hline 1. & DC Geared Motor & 12 Volts ,10rpm \\
\hline 2. & $\begin{array}{ll}\text { HC-05 } & \text { TTL } \\
\text { Module } & \end{array}$ & $\begin{array}{l}9600 \text { bps }, 3.3 \text { to } 5 \mathrm{~V} \\
\mathrm{I} / \mathrm{O}\end{array}$ \\
\hline 3. & Relay & 4 Channel ,5 Volt \\
\hline 4. & Fasteners & - \\
\hline 5. & $\begin{array}{l}\text { Arduino } \\
\text { ATMega328P }\end{array}$ & $\begin{array}{ll}\text { Operating } & \text { voltage } \\
5 \mathrm{~V} \text {,Input } & \text { voltage } \\
7-12 \mathrm{~V} & \end{array}$ \\
\hline 6. & Aluminium flats & - \\
\hline 7. & Solar Panel & $5 \mathrm{~W}, 17.1 \mathrm{~V}$ \\
\hline 8. & $\begin{array}{l}\text { Rechargeable } \\
\text { Battery }\end{array}$ & 12Volts,10ah \\
\hline 9. & $\begin{array}{l}\text { General Purpose } \\
\text { Diode } 1 \mathrm{n} 4004\end{array}$ & $\begin{array}{l}\text { Reverse current } 5 \\
\text { milliampere. }\end{array}$ \\
\hline 10. & Wires & - \\
\hline
\end{tabular}

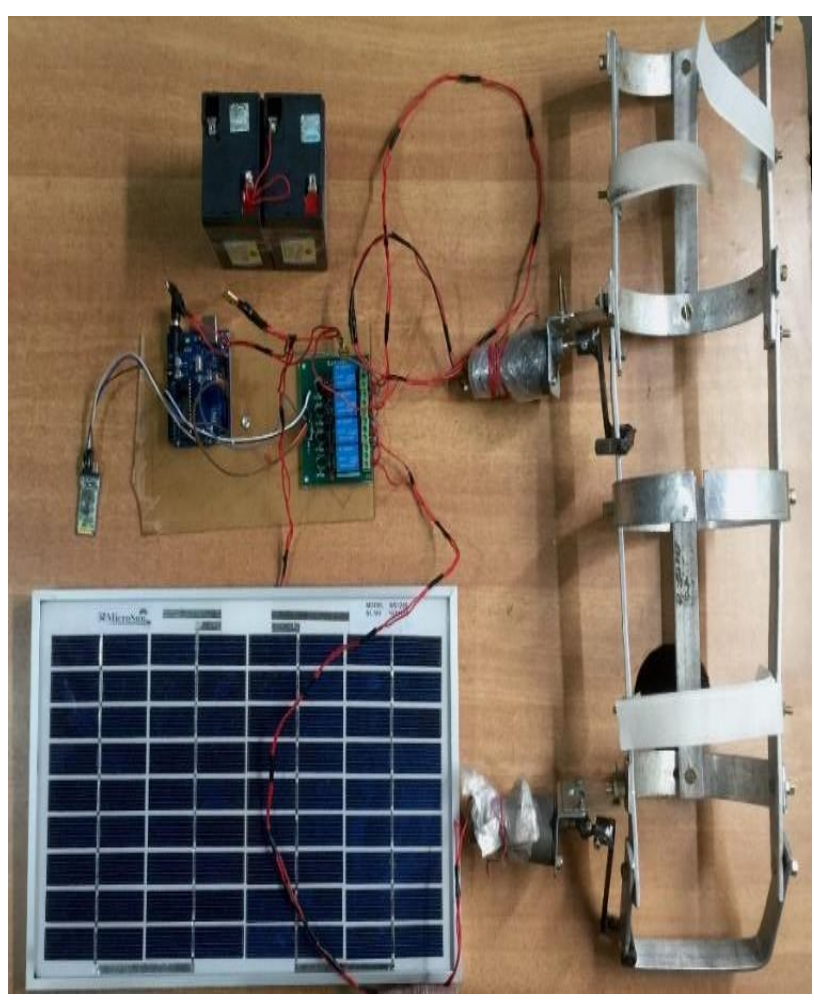

Fig.3. The picture is the complete model of android based voice operated exoskeleton arm using solar power including all components. 
Table.2. The table represents each voice commands used with its corresponding operation and its required time.

\begin{tabular}{|l|l|l|l|}
\hline Sl.No & $\begin{array}{l}\text { Voice } \\
\text { Commands }\end{array}$ & Operation & $\begin{array}{l}\text { Tim } \\
\text { e Re } \\
\text { quir } \\
\text { ed }\end{array}$ \\
\hline 1 & Up & $\begin{array}{l}\text { Wrist } \\
\text { moves } \\
\text { upwards }\end{array}$ & $3 \mathrm{sec}$ \\
\hline 2 & Down & $\begin{array}{l}\text { Wrist moves } \\
\text { downwards }\end{array}$ & $\begin{array}{l}3.4 \\
\text { sec }\end{array}$ \\
\hline 3 & Elbow Up & $\begin{array}{l}\text { Elbow } \\
\text { moves } \\
\text { upwards }\end{array}$ & $6 \mathrm{sec}$ \\
\hline 4 & $\begin{array}{l}\text { Elbow } \\
\text { down }\end{array}$ & $\begin{array}{l}\text { Elbow } \\
\text { moves } \\
\text { downwards }\end{array}$ & $5 \mathrm{sec}$ \\
\hline
\end{tabular}

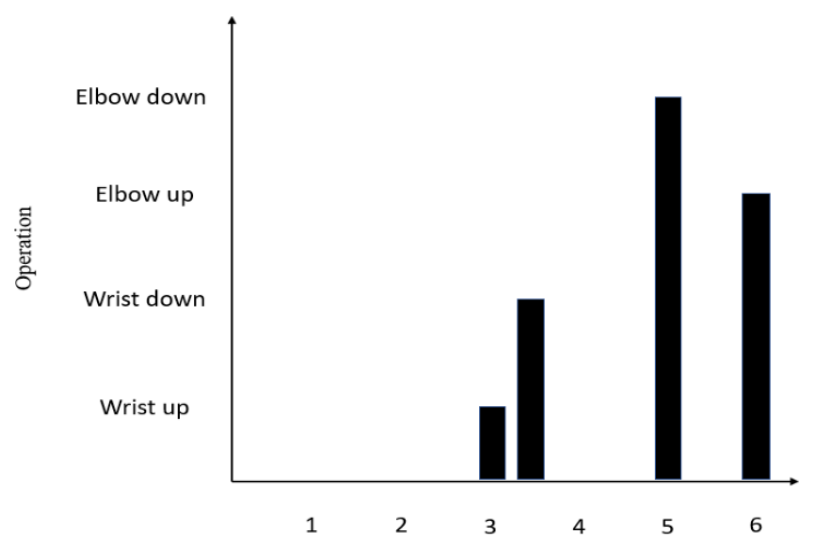

Figure.4. The figure exhibits the graphical representation of time versus operation of the exoskeleton arm.

\section{Conclusions}

It may be seen very well from the overview that a large portion of the propelled work in this field has been done in ongoing decades and a considerable lot of the results have been exhibited in wired situations. Force source innovations and solid remote advancements are utilized, with the goal that it is agreeable for outdoor chores[20]. Ensuring the portability of the arm exoskeleton framework is conceivably the most challenging part of the development. In the review, it is discovered that the emphasis is on a three DOF. The use of android application made the system more reliable by providing a wireless environment. No issue what number of DOFs are included for the exoskeleton, the exoskeleton is benefiting the human[16]. Further survey is required on exoskeleton control framework with the goal that it might understand more about the exoskeleton. The $5 \mathrm{~W}$ solar panel used can be compacted by replacing with $1.3 \mathrm{~W}$ solar panel .Advancement in software program can be introduced and thus time consuming can be reduced. This is a prototype model of exoskeleton arm. When it comes to practical real life sense ,many advancements can be included as per requirements.

\section{References}

[1]. Megalingam, Rajesh Kannan, et al. "Voicebased Hand Orthotic Device." 2019 International Conference on Communication and Signal Processing (ICCSP). IEEE, 2019.

[2]. Suberbiola, Aaron, et al. "Arm orthosis/prosthesis movement control based on surface EMG signal extraction." International journal of neural systems 25.03 (2015): 1550009.

[3]. Gopura, R. A. R. C., Kazuo Kiguchi, and D. S. V. Bandara. "A brief review on upper extremity robotic exoskeleton systems." 2011 6th international Conference on Industrial and Information Systems. IEEE, 2011.

[4]. Hsu, Tzu-Heng, et al. "A finger exoskeleton robot for finger movement rehabilitation." Inventions 2.3 (2017): 12

[5]. Abdallah, Ismail Ben, Yassine outeraa, and chokri rekik. "Design and development of 3d printed myoelectric robotic exoskeleton for hand rehabilitation." international journal on smart sensing \& intelligent systems 10.2 (2017) ventimiglia, paul michael. "design of a human hand prosthesis." (2012).

[6]. Beyrouthy, Taha, et al. "EEG mind controlled smart prosthetic arm." 2016 IEEE International Conference on Emerging Technologies and Innovative Business Practices for the Transformation of Societies (EmergiTech). IEEE, 2016 
[7].Lucas, Lenny, Matthew DiCicco, and Yoky Matsuoka. "An EMG-controlled hand exoskeleton for natural pinching." Journal of Robotics and Mechatronics 16 (2004): 482-4881

[8]. Shankar, Mr SS Azhagu, and Mr Akhil Raj KV. "Design and Fabrication of Voice Activated Robotic Arm." (2017).

[9].Song, Zhibin, et al. "Implementation of resistance training using an upper-limb exoskeleton rehabilitation device for elbow joint." J. Med. Biol. Eng 34.2 (2014): 188- 196

[10]. Mao, Ying, and Sunil Kumar Agrawal. "Design of a cable-driven arm exoskeleton (CAREX) for neural rehabilitation." IEEE Transactions on Robotics 28.4 (2012): 922-931.

[11]. Vaca Benitez, Luis Manuel, et al. "Exoskeleton technology in rehabilitation: Towards an EMG-based orthosis system for upper limb neuromotor rehabilitation." Journal of Robotics 2013 (2013).

[12]. Pons, J. L., et al. "Upper-limb robotic rehabilitation exoskeleton: Tremor suppression." Rehabilitation robotics (2007): 453-470.

[13]. Restrepo-zapata, julio, et al. "twodegree adjustable exoskeleton for assistance of the human arm using a mechanical system of fast assembly and upgradability." international journal on smart sensing \& intelligent systems 10.3 (2017).

[14]. Bouteraa, Yassine, and Ismail Ben Abdallah. "Exoskeleton robots for upperlimb rehabilitation." 2016 13th International Multi-Conference on Systems, Signals \& Devices (SSD). IEEE, 2016.

[15]. DiCicco, Matthew, Lenny Lucas, and Yoky Matsuoka. "Comparison of control strategies for an EMG controlled

[16]. orthotic exoskeleton for the hand." IEEE International Conference on Robotics and Automation, 2004. Proceedings. ICRA'04. 2004. Vol. 2. ieee, 2004.

[17]. Nair, Shyam R., and Shalini R. Nair.
"Design of a Voice Controlled Robotic Arm for Picking and Placing an Object." IOSR Journal of Engineering 2.4 (2012): 670- 673.

[18]. Joshi, Shounak, Ketan Khedkar, and Atharva Kunte. "Design And Fabrication of a Myoelectric Controlled Prosthetic Hand." IJARIIE-ISSN (O)-2395-4396 1.3 (2015).

[19]. Samant, Piyush, and Ravinder Guide Agarwal. Real-time speech recognition system for prosthetic arm control. Diss. 2014.

[20]. Chakarov, D., et al. "New exoskeleton arm concept design and actuation for haptic interaction with virtual objects." Journal of Theoretical and Applied Mechanics 44.4 (2014): 3-14.

[21]. Gopura, R. A. R. C., et al. "Developments in hardware systems of active upper-limb exoskeleton robots: A review." Robotics and Autonomous Systems 75 (2016): 203-220.

[22]. Lo, Ho Shing, and Sheng Quan Xie. "Exoskeleton robots for upper-limb rehabilitation: State of the art and future prospects." Medical engineering \& physics 34.3 (2012): 261-268

[23]. Noda, Tomoyuki, et al. "Development of an upper limb exoskeleton powered via pneumatic electric hybrid actuators with bowden cable." 2014 IEEE/RSJ International Conference on Intelligent Robots and Systems. IEEE, 2014

[24]. Abdallah, ismail ben, yassine bouteraa, and chokri rekik. "design and development of $3 \mathrm{~d}$ printed myoelectric robotic exoskeleton for hand rehabilitation." international journal on smart sensing \& intelligent systems 10.2 (2017)

[25]. "Stretchable materials for robust soft actuators towards assistive wearable devices." Scientific reports 6 (2016): 34224. 